\title{
On the Economic Operation of Regional Power Grid
}

\author{
Wang Ling, Gong Xuejun \\ Benxi Power Supply Company, State Grid Liaoning Electric Power Supply Co., Ltd. \\ bxwl_650928@126.com
}

Keywords: power grid; economic operation; reducing power loss and saving energy

\begin{abstract}
In order to reduce power grid consumption, achieve better management and improve economic efficiency of power grid enterprises, this paper analyzes the technical and management causes for grid losses, proposes technical measures to reasonably manage the economic operation of transformers, optimize reactive voltage, adjust scientifically the loading, optimize economically the operation, structure and equipment of power grid, and management measures to strengthen the calculation of grid loss theoretically and management over users, attempting to provide references to reduce power loss while transmitting the same power and improve the economic operation of regional power grid.
\end{abstract}

Power system is an energy production and consumption system consisting of power generation, transmission, substation, distribution and consumption. Grid enterprises transmit power from power plants to all types of users by series of processes and obtain the price difference of purchasing and selling power as profit. Take the electricity supply of a prefecture-level city 8 billion $\mathrm{kWh}$ as example. A power loss rate of $6 \%$ means an annual power loss of 480 million $\mathrm{kWh}$; and every $0.1 \%$ reduction of power loss would help save 8 million $\mathrm{kWh}$, bringing another 5.2 million yuan of profits on the basis of the average electricity price 0.65 yuan / $\mathrm{kWh}$ in Liaoning Power Grid.

Therefore, under the overall environment of increasing depletion of traditional energy sources and advocating of energy conservation, managers of grid enterprises begin to pay more attention to power loss management, actively implement better management over power loss, explore effective measures to improve scientific management in grid enterprises. This paper mainly attempts to analyze factors leading to power loss and measures to reduce power loss, so as to realize economic operation of power grid on the basis of meeting power need and ensuring the safe operation of grid and power quality

\section{Causes for Power Loss}

Power loss is caused by lines, transformers and other equipment components during the process of transmitting and distributing electric power, including fixed power loss, variable loss and other losses. Fixed loss mainly refers to electricity loss caused by grid equipment like transformer; it changes with the load and is mainly related to the specific capacity, applied voltage on the equipment and quality of the product. Variable loss mainly refers to the electricity loss caused by equipment like lines and transformers in the grid; it changes with the load current and is in proportion to the squared current. Other losses refer to electricity loss caused by poor human management over power supply and consumption, etc.

\section{Technical Factors for Power Loss}

Technical factors for power loss in grid include: unreasonable capacity of transformers and large difference between high and low load rate that make it difficult for transformer to stay in economic operation stage constantly; using transformer and other components of high consumption; low power coefficient due to insufficient reactive power compensation; unbalanced three-phase load; large difference between peak and valley loads; improper operation mode, operation voltage and structure of power grid.

\section{Management Factors for Power Loss}

Management factors for power loss mainly include: insufficient optimization of reactive power voltage; absence of a sound assessment and incentive mechanism in management over economic 
dispatch in power grid; failure in eliminating or improving technically transformer and other equipment of high consumption; imperfect management and assessment system over power stealing by users that makes it unable to reduce lines and areas of high consumption effectively; failure in guiding load demand by differentiating the prices of electricity at peak and valley.

\section{Measures to Reduce Power Loss}

\section{Economic Operation of the Transformer}

Power loss caused by the transformer accounts for nearly $50 \%$ and thus the economic operation of transformer is of great significance for reducing power loss. Power loss in transformer consists of no-load loss (iron loss) and load loss (copper loss). As we all know, transformer has the highest efficiency and minimum consumption when operating in the best economic point (iron loss equals to copper loss), where the capacity of transformer doesn't play to the fullest. Therefore, in practice, it is necessary to manage the operation of transformer according to the economic operation point and capacity-load ratio of the transformer, so as to reduce consumption of power grid and at the same time achieve the maximum efficiency of the transformer.

This paper takes two-winding transformer as example to make deep analysis over the economic operating zone of transformer. It classifies the operating zone of transformer into uneconomic operating zone an economic operating zone and proposes the optimum operating zone of the transformer, attempting to provide references for operators of transformer to manage the operation of transformer scientifically.

\section{Optimizing Reactive Voltage}

Coefficient of grid capacity directly affects the amount of power loss. Therefore, we should minimize the long-distance transmission of reactive power in the power grid and adopt the combination of centralized compensation and dispersion compensation to install compensation equipment of appropriate capacity at the right position according to the principle of "layering and zoning, balance on the spot", so as to achieve the goal of increasing operating voltage and reducing power loss.

Sufficient reactive power supply is the prerequisite for ensuring the quality and economy of voltage in the grid. The gradual advancement of unmanned substation puts forward higher requirements on the automation level of voltage regulation. We should increase the on-load voltage regulating rate of the transformer, choose reactive compensation equipment of reasonable capacity, promote the implementation of AVC technology, improve the optimization of grid and thus realize the optimized management over grid voltage and power loss by scientific control over transformer taps and capacitor switching according to the changing law of the load. In order to achieve management over grid power loss by reactive voltage, it is necessary to take scientific measures to keep reactive voltage under control.

Reverse voltage regulation is commonly used in power grid. It requires increasing the voltage at key buses under the maximum load to compensate for the increased voltage consumption of the line because of the maximum load; it requires reducing the voltage at key buses under the minimum load to prevent the voltage at the load point from being too high and ensure economic operation of the grid. Capacity loss in the line and windings of the transformer is inversely proportional to the square of the voltage, while the iron loss of the transformer is proportional to the square of the voltage. Operating voltage can be increased properly if the former accounts for a large part of the total losses. Generally, operating voltage can be increased properly to reduce losses in grid of $35 \mathrm{kV}$ and above. For distribution network of $10 \mathrm{kV}$, the no-load loss accounts for about $50 \%$ to $80 \%$ of the total losses and especially the proportion of no-load losses is much larger at night. Therefore, operating voltage can be reduced properly to decrease power loss effectively.

\section{Regulating the Voltage Scientifically}

The more imbalanced the three-phase load in the grid, the greater the power loss. Therefore, it is generally required to keep the imbalance rate of three-phase current under low voltage in power transformer no more than $10 \%$ and the imbalance rate of current at the main line of low voltage no more than $20 \%$. A large imbalance rate of three-phase voltage means that losses of the line and 
transformer increase and that the safe operation of the equipment would be endangered and needing in-time regulation. Therefore, the three-phase load data should be regularly monitored and analyzed and management over the load of power consumption side should be strengthened to make three-phase loads symmetrical to reduce power losses.

Regulate reasonably the load, increase the load rate of power grid and reduce the difference between peak and valley loads. Under the condition of consuming the same amount of electricity, the higher the load rate and the smaller the difference between peak and valley loads, the less the power loss. Therefore, power loss can be effectively reduced by regulate reasonably the distribution of load and improving load rate. From the perspective of the grid, the operator should strengthen load forecasting, distribute the load reasonably and improve the load rate of power supply in the grid. From the perspective of the users, the objective of reducing power loss can be realized by implementing TOU price policy, advocating users to consume power at the valley period, keeping balance between peak-load and off-peak load and increasing load rate.

\section{Optimizing the Operating Mode of the Grid}

The economic operation of the grid is a complex and systematical project that should be analyzed and regulated from the perspective of the whole network. The economic operating mode of the grid should meet the requirement of minimizing power loss on the basis of satisfying power demand and ensuring safe operation of the grid and quality of the supplied power. Adjusting the operating mode of the grid is the most reasonable measure to reduce power loss. Economic operating mode of the grid generally consists of distributing loads according to the optimized power flow, manage reasonably the transformer, regulate the operating voltage scientifically, increase the capacity coefficient of reactive compensation, adjust the loads and scientifically arrange equipment maintenance plan, etc.

Arrange maintenance work reasonably. Power losses during maintenance of electrical equipment and implementation of new equipment are much larger than under normal operation since there are always changes in the operating mode of the grid then. Therefore, it is necessary to strengthen management over maintenance work arrangement, optimize operating mode and maintenance plan of the grid, minimize power outages and avoid unplanned and repeated blackouts, cooperate with users to finish maintenance work during the minimized period of time, promote live maintenance work mode actively and choose from several alternative maintenance plans under the condition of ensuring security of power supply, so as to reduce the power loss during maintenance of the grid.

Increase the capacity of a $220 \mathrm{kV}$ substation supplying power for a county-level city from120MVA into 180MVA with an outage duration of 30 days and a load of $66 \mathrm{kV}$ to be reasonably distributed. Solution 1: transfer all the load of this substation by another main transformer. Solution 2: transfer part of the load in this substation by another main transformer through a main line, where part of the load is transferred by a $66 \mathrm{kV}$ tie line. Solution 3: transfer the load of the replaced transformer by another main transformer through a main line, where part of the load is transferred by a $66 \mathrm{kV}$ tie line and part of the load is transferred into the adjacent system. Solution 3 is chosen according to the State Council Decree 599 Regulation on the Emergency Response to and Investigation and Handling of Electric Power Safety Accidents and requirements of economic operation of the grid. From the perspective of security, line wire section allows the load current to meet the requirements of load carried; relay protection and automatic safety devices are rearranged to avoid serious large-scale blackouts when an accident occurs. From the perspective of economic operation, the loads are distributed evenly to prevent transformer and lines from being heavily loaded or overloaded; voltage of the nodes can meet the requirements and the power flow is arranged reasonably.

\section{Conclusion}

Economic operation of the grid adopts very practical energy-saving technology to make full advantage of the existing equipment and components without or with little investment under the premise of ensuring security of power supply and power quality in the grid by technical measures, 
including economic operation of the transformer, optimizing reactive voltage, increasing capacity coefficient, adjusting voltage, optimizing the operating mode of the grid, optimizing the structure and equipment of the grid, and necessary supplementing management measures to realize the objective of reducing power loss in the system and increasing economic efficiency for the grid enterprises while transmitting the same amount of electricity.

\section{References}

[1] Human Resources Department of State Grid Corporation, power system (Analysis) [M] Beijing: China Electric Power Press, 2010.

[2] Qin Yun, Zhang Sihan. Analysis and Measures for Economic Operation of the Grid [J] Heilongjiang Electric Power, 2009 (05): 334-337.

[3] Li Penghui, Liu Yitao. Determination and Application of the Economic Operation Zone of the Transformer [J] Demand Side Management, 2010 (04): 28-31.

[4] Ye Liangfeng, Di Daoguo. Analysis and Measures for Economic Operation of the Grid [J]. Guangxi Electric Power, 2005 (4): 71-73.

[5] Power Industry Occupational Skill Testing Guidance Center, substation attendant [M] .2nd edition, Beijing: China Electric Power Press, 2008.

[6] Hou Baosu, Wei Suqin, Lei Yuqing. Analyzing the Line Loss in Regional Grid and Exploring Countermeasures [C] 2011 Proceedings of academic meetings of the Asia Pacific Smart Grid and Information Engineering, 2011: 125-128.

[7] Zhao Caihong, Tang Yinsheng. Several Key Issues in Power Loss Reducing of the Grid in China [J] China Power, 2007 (12): 64-67.

[8] Hou Guangsong, Xie Guangjian. Analysis and Application of Economic Operation of the Regional Grid [J] Shandong Power Technology, 2004 (01): 78-80. 